\title{
Dosimetric comparison of computed tomography- guided iodine-125 seed implantation assisted with and without three-dimensional printing non-coplanar template in locally recurrent rectal cancer: a propensity score matching study
}

\author{
Lu Wang*, MB, Hao Wang*, MD, Yuliang Jiang, MM, Zhe Ji, MD, Fuxin Guo, MD, Ping Jiang, MD, Bin Qiu, MD, \\ Haitao Sun, MM, Jinghong Fan, MB, Weiyan Li, MB, Junjie Wang, MD, PhD \\ * Lu Wang and Hao Wang contributed equally to this work and are co-first authors of the article.
}

Department of Radiation Oncology, Peking University Third Hospital, Beijing, People's Republic of China

\begin{abstract}
Purpose: To compare post-implant dosimetric parameters of computed tomography (CT)-guided radioactive iodine-125 ( $\left.{ }^{125} \mathrm{I}\right)$ seed (RIS) implantation assisted with and without three-dimensional printing non-coplanar template (3D-PNCT) in locally recurrent rectal cancer (LRRC).

Material and methods: One hundred and fifty-five LRRC patients treated by CT-guided RIS implantation assisted with or without 3D-PNCT from October 2003 to May 2019 were included in this study. Propensity score matching (PSM) method $(1: 1)$ was used to adjust for differences between the 3D-group (with 3D-PNCT) and the CT-group (without 3D-PNCT). After PSM, dosimetric parameters $\left[\mathrm{D}_{90}\right.$ (dose that covered $90 \%$ of target volume), $\mathrm{D}_{100}$ (dose that covered $100 \%$ of target volume), $V_{100}$ (percentage of gross tumor volume (GTV) receiving $100 \%$ of prescription dose), $\mathrm{V}_{150}$ (percentage of GTV receiving $150 \%$ of prescription dose), HI (homogeneity index), CI (conformity index), and EI (external index)] of the two groups were compared.

Results: After PSM, 45 pairs of matched cases were selected for analysis and differences in variables between the two groups were balanced. For the 3D-group, median values of $\mathrm{D}_{90}, \mathrm{D}_{100}, \mathrm{~V}_{100}, \mathrm{~V}_{150}, \mathrm{EI}$, and HI were 142.6 Gy (73.7218.2 Gy), 73.7 Gy (26.2-169.3 Gy), 94.1\% (74.3-100\%), 71.8\% (35.4-98.3\%), 0.7 (0.1-30.7), and 0.20 (0-0.60), respectively, and corresponding values were 119.9 Gy (39.8-159.3 Gy), $47.0 \mathrm{~Gy}$ (13.0-200.9 Gy), 89.9\% (38.6-100\%), 62.8\% (14.8-100\%), 0.39 (0-11.01), and $0.30(0-0.95)$, respectively, for the CT-group. Parameters including $\mathrm{D}_{90}, \mathrm{D}_{100}, \mathrm{~V}_{100}, \mathrm{~V}_{150}$, and EI in the 3D-group were significantly higher than those in the CT-group $(p<0.001, p<0.001, p<0.001, p<0.001$, and $p=0.006$, respectively).
\end{abstract}

Conclusions: 3D-PNCT can improve the accuracy of radioactive seed implantation by increasing the dose delivered to the tumor and reducing the number of "cold" spots of dose.

J Contemp Brachytherapy 2021; 13, 1: 18-23 DOl: https://doi.org/10.5114/jcb.2021.103582

Key words: 3D printing template, seed implantation, dosimetry, locally recurrent rectal cancer.

\section{Purpose}

Radioactive iodine-125 ( $\left.{ }^{125} \mathrm{I}\right)$ seed (RIS) implantation has the advantages of delivering a high-dose in the target volume, sharp dose fall-off around the tumor, and a lower amount of irradiation to the surrounding organs at risk (OARs). The safety and efficacy of RIS have been confirmed, and RIS is widely used in the treatment of various tumors, including prostate cancer [1], head and neck cancer [2], lung cancer [3], pancreatic cancer [4], and rectal cancer [5]. The therapeutic effect of RIS implantation depends on the spatial distribution of dose, which is in turn dependent on the distribution of implantation needles (needle position, direction, and depth) and the arrangement of seeds. Therefore, it is essential to improve the accuracy of RIS implantation. The efficacy of free-
Address for correspondence: Junjie Wang, MD, PhD, Department of Radiation Oncology, Peking University Third Hospital, 49 North Garden Road, Haidian District, Beijing, 100191, People's Republic of China, phone: +86-010-82265921, fax: +86-010-62017700, ₫ e-mail: junjiewang@pku.edu.cn
Received: 31.07 .2020

Accepted: 31.10 .2020

Published: 18.02.2021 
hand computed tomography (CT)-guided RIS implantation is predominantly influenced by operator experience, complex anatomical structure, and irregular borders of the tumor. To further improve the accuracy of RIS implantation, Huang et al. were the first to employ three-dimensional printing non-coplanar template (3D-PNCT) for CT-guided RIS implantation in the treatment of head and neck tumor [6], and their results were proved safe, effective, and accurate $[7,8,9]$. However, few studies have compared dosimetric parameters of CT-guided RIS implantation assisted with and without 3D-PNCT. In this study, the differences in the dosimetric parameters of patients with locally recurrent rectal cancer (LRRC) treated by CT-guided RIS implantation with (3D-group) and without 3D-PNCT (CT-group) were investigated using propensity score matching method.

\section{Material and methods}

\section{Patient enrollment and clinical characteristics}

Data of 155 LRRC patients who were treated by CT-guided RIS implantation with or without 3D-PNCT in our department from October 2003 to May 2019 were retrospectively analyzed. This study was approved by the Institutional Ethics Committee of our hospital (No. IRB00006761). All patients selected for RIS implantation met the following criteria: 1) pathologically diagnosed as LRRC, 2) extraluminal pelvic recurrence, without distal metastasis or with controllable oligometastasis, 3) tumor diameter $\leq 7 \mathrm{~cm}, 4$ ) suitable puncture access, 5) no bleeding tendency, 6) Karnofsky performance status $>70$, 7) life expectation $\geq 3$ months. The patients began to be treated by CT-guided RIS implantation with 3D-PNCT when the technology was introduced into our department in 2015. The clinical characteristics of all patients are shown in Table 1.

\section{Treatment procedures}

\section{Pre-operative CT localization and planning design}

All patients underwent spiral CT two days before seed implantation. The prone or supine position was chosen according to the tumor site. Next, the patients were immobilized with vacuum pads, and their positioning lines were marked on skin surface. The CT data were transmitted to a brachytherapy treatment planning system (B-TPS). Then, oncologists and physicists designed the pre-operative plan, which included drawing of gross tumor volume (GTV) and OARs, setting of prescription dose and RIS activity, design of needle pathways, simulation of spatial seed distribution, and verification of dosimetric parameters.

\section{Intra-operative procedures}

CT-guided RIS implantation without 3D-PNCT assistance - the CT-group: Before December 2015, 91 patients underwent CT-guided RIS implantation without 3D-PNCT assistance. After local or spinal anesthesia, needles were inserted into the tumor and arranged in parallel with $1 \mathrm{~cm}$ intervals, extending $0.3-0.5 \mathrm{~cm}$ beyond the margin of the tumor. Then, the seeds were implanted with a Mick seed implantation gun and were spaced 0.5-1.0 cm apart.

CT-guided RIS implantation assisted with 3D-PNCT - the 3D-group: From December 2015, individualized 3D-PNCT was utilized, and 64 patients were treated with CT-guided RIS implantation assisted by 3D-PNCT. A 3D rapid prototyping equipment printed the individualized 3D-PNCT with photo-curable resins according to the pre-operative design. The template contained the superficial anatomic characteristics of treatment area, needle pathways, and positioning markers. After anesthesia, we applied the 3D-PNCT on the pre-planned area according to the positioning markers. Then, the needles were inserted with the planned depth through the needle pathways in the temple. The seeds were implanted with a Mick gun according to the pre-operative plan (Figures 1 and 2).

\section{Post-operative dosimetry verification}

All patients underwent a CT scan immediately after seed implantation to verify actual spatial distribution of seeds and to calculate dosimetric parameters. The dosimetric parameters were as follows: $\mathrm{D}_{90}$ (dose that covered $90 \%$ of target volume), $\mathrm{D}_{100}$ (dose that covered $100 \%$ of target volume), $V_{100}$ (percentage of GTV receiving $100 \%$ of prescription dose), $V_{150}$ (percentage of GTV

Table 1. Clinical characteristics of all patients

\begin{tabular}{lc} 
Characteristics & Value \\
\hline Sex & 103 \\
\hline Male & 52 \\
\hline Female & $57.1 \pm 11.3$ \\
\hline Age (years), average \pm standard deviation & 151 \\
\hline Histopathology & 1 \\
\hline Adenocarcinoma & 3 \\
\hline Squamous carcinoma & 3 \\
\hline Adenosquamous carcinoma & 124 \\
\hline Previous surgery & 27 \\
\hline None & 1 \\
\hline Once & 79 \\
\hline Twice & 48 \\
\hline Three times & 17 \\
\hline Cumulative dose in the pelvis, EQD & (Gy) \\
\hline$<50$ & 11 \\
\hline $50-100$ & 13 \\
\hline$>100$ & 112 \\
\hline Unclear & 26 \\
\hline Courses of previous radiotherapy & $48.1(1.8-297.5)$ \\
\hline 0 & \\
\hline 1 & \\
\hline 2 & \\
\hline 3 & \\
\hline GTV (ml), median (range) & \\
\hline
\end{tabular}

$E Q D_{2}$ - equivalent dose in $2 \mathrm{~Gy} / f x, G T V$ - gross tumor volume 


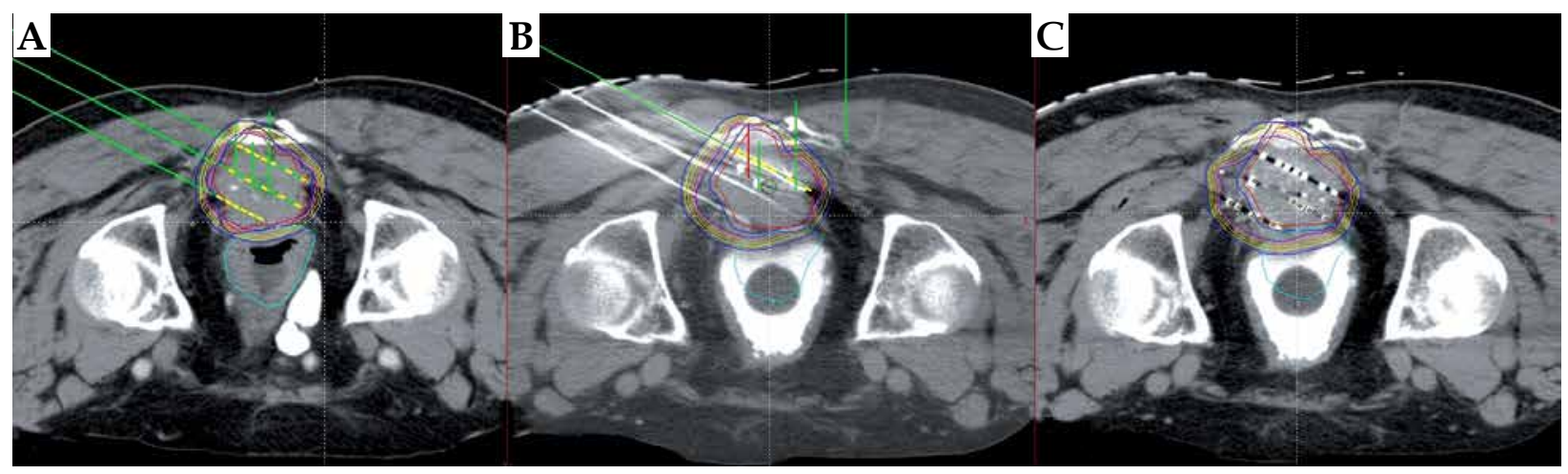

Fig. 1. Pre-operative, intra-operative, and post-operative computed tomography (CT) images from CT-guided radioactive ${ }^{125}$ I seed (RIS) implantation assisted with three-dimensional printing non-coplanar template (3D-PNCT), respectively (left to right). A) The pre-operative plan in the brachytherapy treatment planning system (B-TPS), B) the actual position of needles during the operation, C) the distribution of RIS after implantation
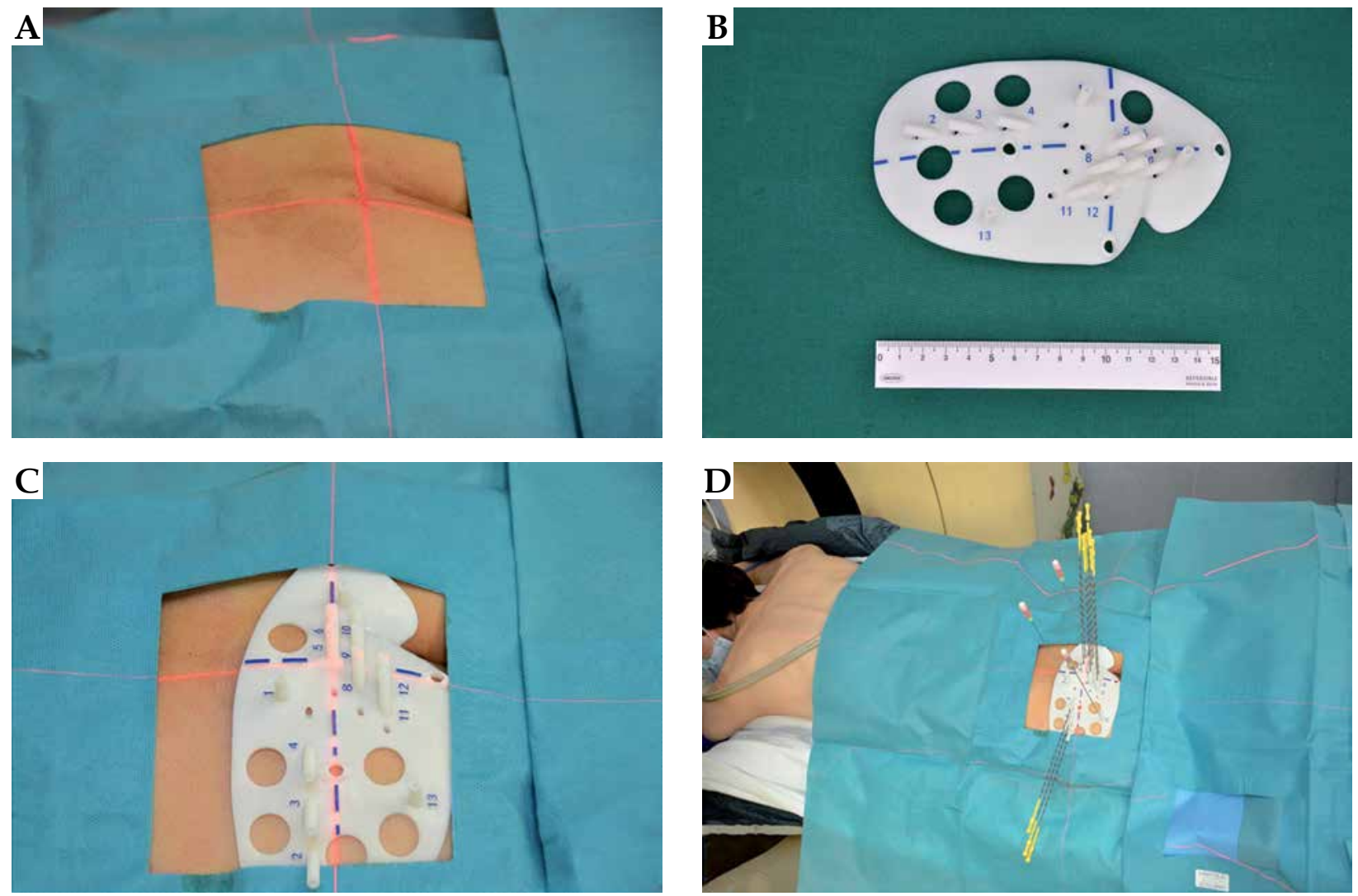

Fig. 2. The procedure of computed tomography (CT)-guided radioactive ${ }^{125}$ I seed (RIS) implantation assisted with three-dimensional printing non-coplanar template (3D-PNCT). A) The pre-operative CT localization. The positioning lines were marked on the skin surface according to the laser lines, B) the 3D-PNCT that fitted the anatomic characteristics of treatment area on the skin surface and contained the information of needle pathways (position, direction, and depth), C) the 3D-PNCT fixed on the skin surface according to the positioning lines, D) the needles inserted into the planned depth through needle pathways in the 3D-PNCT

receiving $150 \%$ of prescription dose), homogeneity in$\operatorname{dex}[10]$ (HI: the homogeneity of dose distribution, $\mathrm{HI}=$ $\left(\mathrm{V}_{\mathrm{T}, \text { ref }}-\mathrm{V}_{\mathrm{T}, 1.5 \mathrm{ref}}\right) / \mathrm{V}_{\mathrm{T}, \text { ref, where }} \mathrm{V}_{\mathrm{T} \text {, ref, }}$ and $\mathrm{V}_{\mathrm{T}, 1.5 \mathrm{ref}}$ were the volume of GTV receiving prescription dose and the volume of GTV receiving $150 \%$ of the prescription dose $\left(\mathrm{cm}^{3}\right)$, respectively), conformity index [11] (CI: the con- formity of dose distribution, $\mathrm{CI}=\left(\mathrm{V}_{\mathrm{T}, \text { ref }} / \mathrm{V}_{\mathrm{T}}\right) \times\left(\mathrm{V}_{\mathrm{T} \text {, ref }} /\right.$ $\mathrm{V}_{\text {ref }}$ ), where $\mathrm{V}_{\mathrm{T}}$ and $\mathrm{V}_{\text {ref }}$ were the volume of GTV and the total volume receiving prescription dose $\left(\mathrm{cm}^{3}\right)$, respectively), external index [10] (EI: the percentage of volume outside GTV that received exceeded prescription dose, $\left.\mathrm{EI}=\left(\mathrm{V}_{\text {ref }}-\mathrm{V}_{\mathrm{T}, \mathrm{ref}}\right) / \mathrm{V}_{\mathrm{T}}\right)$. 


\section{Statistical analysis}

All the analyses were carried out using SPSS version 22.0 (IBM Corp., Armonk NY, USA). Normally distributed data were expressed as mean \pm standard deviation, and non-normally distributed data were expressed as median and range. Categorical data were analyzed with $\chi^{2}$ test or Fisher's exact test. Continuous data were evaluated using unpaired $t$-test for normally distributed data and Mann-Whitney $U$ test for non-normally distributed data. Propensity scores were estimated with a logistic regression model to control the potential bias. Matching covariates included age, gender, GTV, and tumor location [12]. The 3D-group and CT-group were matched using a $1: 1$ nearest neighbor matching method, with a caliper lower than 0.1 . The adequacy of the matching procedure was assessed with a standardized difference (StDiff). A difference of $<10 \%$ were considered well-balanced [13]. The differences in the post-operative dosimetric parameters between the matched groups were further compared. $P$-value $<0.05$ was considered statistically significant. All $p$-values were two-sided.

\section{Results}

A total of 155 patients were enrolled in this study. The patients were divided into two groups (3D-group and CT-group). Variables, including age, gender, GTV, and tumor location were enrolled in propensity score matching (PSM). After PSM with a $1: 1$ ratio, both 3D-group and CT-group comprised 45 patients. No significant differences were observed in age $(p=0.673)$, gender $(p=0.480)$, GTV $(p=0.493)$, and tumor location $(p=0.953)$. The baseline characteristics of the two groups before and after PSM are presented in Table 2.

After PSM, baseline characteristics balanced overall between the two groups, except for male (3D-group, $75.6 \%$ vs. CT-group, $68.9 \%$; StDiff, $13.4 \%$ ). The non-parametric test showed that values of $\mathrm{D}_{90}, \mathrm{D}_{100}, \mathrm{~V}_{100}, \mathrm{~V}_{150}$, and EI of 3D-group were significantly higher than those of CT-group $(p<0.001, p<0.001, p<0.001, p<0.001$, and $p=0.006$, respectively) (Table 3 ). The value of HI in CTgroup was significantly higher than that of $3 \mathrm{D}$-group $(p=0.001)$. For the 3D-group, the median values of $\mathrm{D}_{90}$, $\mathrm{D}_{100}, \mathrm{~V}_{100}, \mathrm{~V}_{150}$, EI, and HI were 142.6 Gy (73.7-218.2 Gy), 73.7 Gy (26.2-169.3 Gy), 94.1\% (74.3-100\%), 71.8\% (35.4$98.3 \%), 0.7(0.1-30.7)$, and $0.20(0-0.60)$, respectively, and the corresponding values were 119.9 Gy (39.8-159.3 Gy), 47.0 Gy (13.0-200.9 Gy), 89.9\% (38.6-100\%), 62.8\% (14.8$100 \%), 0.39(0-11.01)$, and $0.30(0-0.95)$, respectively, for CT-group. No significant difference was observed in $\mathrm{CI}$ and total activity of seeds $(p=0.394$ and $p=0.771$, respectively).

\section{Discussion}

Free-hand CT-guided seed implantation has many disadvantages. First, the accuracy of free-hand implantation relies on the experience of operators. Post-operative dosimetric parameters can be inconsistent with pre-operative planned parameters. Any misplaced seed can lead to unsatisfactory efficacy and even severe adverse effects. Second, during the implantation, the position, direction, and depth of implantation needles need to be adjusted many times until the distribution of needles is optimal, and the patient needs to undergo multiple CT scans. The efficiency is low, and the patient's risk of complications increases. Last but not least, the complex anatomy of pelvis and irregular borders of the tumor may increase the difficulty of inserting implantation needles.

To further improve the accuracy of seed implantation, 3D-PNCT was combined with CT-guided RIS implantation for tumor treatment. Huang et al. [6] firstly studied CT-guided RIS implantation assisted with 3D-PNCT in 31 head and neck cancer patients, and $\mathrm{D}_{90}, \mathrm{~V}_{100}$, and $\mathrm{V}_{150}$ of these patients met the treatment requirements adequately, confirming that 3D-PNCT can make RIS implantation more accurate and efficient. Individualized 3D-PNCT is designed according to pre-operative plan. It can fit the anatomic characteristics of treatment area on the skin surface and contains information about needle pathways. Many studies have revealed that 3D-PNCT can ensure consistency between pre-operative and post-operative dosimetric parameters $[14,15,16]$. Ji et al. [14] studied 14 cases of different tumors treated by 3D-PNCT-assisted CT-guided RIS implantation. Pre-operative and post-operative values of $D_{90}, V_{100}, V_{150}, V_{200}$, HI, EI, and CI showed no difference. Liu et al. [9] investigated 16 patients with brain gliomas, and no difference was found between pre-operative and post-operative parameters. Besides, some scholars further confirmed that CT-guided RIS implantation assisted with 3D-PNCT was safe and effective. Ji et al. [16] compared the parameters between 3D-PNCT plan and 3D printing coplanar template (3D-PCT) plan to treat peripheral lung cancer. This study revealed that although the dosimetric parameters were similar between the two plans, the 3D-PNCT plan delivered a higher dose in the target volume margin, requiring fewer needles and causing less damage. Wang et al. [17] studied 66 patients with LRRC who were previously treated with EBRT or surgery. Median overall survival (OS) and local control (LC) time were 14.7 and 12.2 months, respectively. Pain relief was achieved in $85.1 \%$ of patients, and $9.1 \%$ of patients had $\geq$ grade 3 side effects.

However, there are few studies about the differences between CT-guided RIS implantation assisted with 3D-PNCT and free-hand CT-guided seed implantation. Han et al. [18] compared the differences of dosimetric parameters between RIS implantation assisted with 3D-PNCT and without 3D-PNCT in treating liver tumors. The results showed that $D_{100}, V_{100}$, and $V_{150}$ values were significantly different between the two groups. $\mathrm{D}_{100}$ and $\mathrm{V}_{100}$ of the template-assisted group were higher, while its $\mathrm{V}_{150}$ was lower than that in the group without a temple auxiliary. In the present study, the $\mathrm{D}_{90}, \mathrm{D}_{100}$, and $\mathrm{V}_{100}$ in the 3D-group were higher than those in the CT-group. Although no significant difference was found in seed number and total activity of seeds, the dose delivered to the 3D-group was higher than that delivered in the CT-group. The median $\mathrm{D}_{90}$ and $\mathrm{V}_{100}$ in 3D-group were 146.2 Gy and 94.1\%, respectively. Previous studies on 
Table 2. Baseline characteristics balance before and after propensity score matching (PSM)

\begin{tabular}{|c|c|c|c|c|c|c|c|c|}
\hline \multirow[t]{2}{*}{ Index } & \multicolumn{4}{|c|}{ Before PSM } & \multicolumn{4}{|c|}{ After PSM } \\
\hline & $\begin{array}{l}\text { 3D-group } \\
(n=64)\end{array}$ & $\begin{array}{l}\text { CT-group } \\
(n=91)\end{array}$ & $P$-value & StDiff (\%) & $\begin{array}{l}\text { 3D-group } \\
(n=45)\end{array}$ & $\begin{array}{l}\text { CT-group } \\
(n=45)\end{array}$ & $P$-value & StDiff (\%) \\
\hline $\begin{array}{l}\text { Age (in years), average } \\
\pm \text { standard deviation }\end{array}$ & $55.1 \pm 10.0$ & $58.4 \pm 12.1$ & 0.079 & 32.6 & $57.5 \pm 9.7$ & $56.6 \pm 11.1$ & 0.673 & 9.4 \\
\hline Male, $n(\%)$ & $37(57.8)$ & $66(72.5)$ & 0.056 & 29.6 & $34(75.6)$ & $31(68.9)$ & 0.480 & 13.4 \\
\hline $\begin{array}{l}\text { GTV }(\mathrm{ml}) \text {, } \\
\text { median (range) }\end{array}$ & $\begin{array}{c}30.0 \\
(1.8-297.5) \\
\end{array}$ & $\begin{array}{c}65.8 \\
(6.5-234.8)\end{array}$ & $<0.001$ & 50.1 & $\begin{array}{c}44.1 \\
(1.8-297.5) \\
\end{array}$ & $\begin{array}{c}57.8 \\
(6.5-234.8) \\
\end{array}$ & 0.493 & 7.5 \\
\hline Location*, n (\%) & & & 0.280 & & & & 0.953 & \\
\hline Sacral invasive type & $31(48.4)$ & $55(60.4)$ & & 23.8 & $24(53.3)$ & $24(53.3)$ & & 0.0 \\
\hline Lateral invasive type & $16(25.0)$ & $20(22.0)$ & & 6.9 & $12(26.7)$ & $11(24.5)$ & & 5.1 \\
\hline Localized type & $17(26.6)$ & $16(17.6)$ & & 20.2 & $9(20.0)$ & $10(22.2)$ & & 5.0 \\
\hline
\end{tabular}

PSM - propensity score matching, StDiff-standardized difference, GTV-gross tumor volume, *sacral invasive type: invasion of lower sacrum (S3, S4, S5), coccyx or periosteum; lateral invasive type: invasion of upper sacrum (S1, S2), sciatic nerve, greater sciatic foramen, or lateral pelvic wall; localized type: invasion of surrounding pelvic organs or tissues [12]

Table 3. Comparison of dosimetric parameters between 3D-group and CT-group after propensity score matching (PSM)

\begin{tabular}{lcccc} 
Parameters & $\begin{array}{c}\text { 3D-group } \\
\text { median (range) }\end{array}$ & $\begin{array}{c}\text { CT-group } \\
\text { median (range) }\end{array}$ & $t / \chi^{2} /$ Mann-Whitney $U$ & $P$-value \\
\hline $\mathrm{D}_{90}(\mathrm{~Gy})$ & $142.6(73.7-218.2)$ & $119.9(39.8-159.3)$ & 444.5 & $<0.001$ \\
\hline $\mathrm{D}_{100}(\mathrm{~Gy})$ & $73.7(26.2-169.3)$ & $47.0(13.0-200.9)$ & 391.5 & $<0.001$ \\
\hline $\mathrm{V}_{100}(\%)$ & $94.1(74.3-100)$ & $89.9(38.6-100)$ & 422.5 & $<0.001$ \\
\hline $\mathrm{V}_{150}(\%)$ & $71.8(35.4-98.3)$ & $62.8(14.8-100)$ & 516.0 & $<0.001$ \\
\hline $\mathrm{HI}$ & $0.20(0-0.60)$ & $0.30(0-0.95)$ & 553.0 & 0.001 \\
\hline $\mathrm{El}$ & $0.7(0.1-30.7)$ & $0.39(0-11.01)$ & 638.5 & 0.006 \\
\hline $\mathrm{Cl}$ & $0.5(0-0.8)$ & $0.59(0.08-0.80)$ & 852.5 & 0.336 \\
\hline Seed number & $66(23-175)$ & $67(6-131)$ & 907.0 & 0.394 \\
\hline Total activity of seeds & $37.8(12.7-119.0)$ & $44.9(2.9-98.3)$ & 976.5 & 0.771
\end{tabular}

$D_{90}$-dose that covered $90 \%$ of target volume, $D_{100}$ - dose that covered $100 \%$ of target volume, $V_{100}$ - percentage of GTV receiving $100 \%$ of prescription dose, $V_{150}$ - percentage of GTV receiving $150 \%$ of prescription dose, $\mathrm{HI}$ (homogeneity index) - homogeneity of dose distribution, $\mathrm{Cl}$ (conformity index) - conformity of dose distribution, El (external index) - percentage of the volume outside GTV that received exceeded prescription dose

prostate cancer suggested that the prognosis of patients with $\mathrm{D}_{90}>140$ Gy and $\mathrm{V}_{100}>90 \%$ was significantly better compared to patients with $\mathrm{D}_{90}<140 \mathrm{~Gy}$ or $\mathrm{D}_{100}<90 \%$ $[19,20,21]$. A study on LRRC revealed that patients with $\mathrm{D}_{90}>130 \mathrm{~Gy}$ or $\mathrm{V}_{100}>90 \%$ had a superior prognosis [17]. One-year local control rates for patients with $\mathrm{D}_{90}>130 \mathrm{~Gy}$ and $\mathrm{V}_{100}>90 \%$ were $72.4 \%$ and $69.3 \%$, respectively. Dosimetric parameters of CT-guided RIS implantation assisted with 3D-PNCT are closer to the optimal values than those of free-hand CT-guided RIS implantation. The "cold" spots of dose can be effectively avoided to improve local control. In the present study, the values of $\mathrm{V}_{150}$ and EI were significantly higher in the 3D-group, whereas the value of HI was significantly lower in this group of patients. The value of CI showed no difference between the two groups. These results suggested that the 3D-group had more "hot" spots of dose inside the tumor than the CT-group. The dose distribution of the CT-group was more homogeneous than that of the 3D-group. In RIS implantation for prostate cancer, Ling et al. [22] investigated dose heterogeneity in terms of radiobiological effect. The authors found that high doses within a heterogeneous pattern contributed to additional tumor cell kill. Herein, it was considered that higher $\mathrm{V}_{150}$ may lead to superior local control of tumor. Dose limit of RIS implantation for small intestine still remains unclear. In this study, the dose limits of OARs referred to the dose limits for prostate RIS implantation and hypofractionated radiotherapy $[23,24]$. A previous study on free-hand CT-guided seed implantation for LRRC showed mild adverse effects, and no severe adverse effects occurred in the small intestine. Therefore, the small intestine dose limit was further decreased in the 3D-group, which could explain a higher EI value in the 3D-group. Although the 3D-PNCT could improve the accuracy of implantation, there were still some errors. The movement of small intestine and colorectum may prevent the needles from being inserted as planned. Moreover, the changes in bladder filling could cause slight changes in tumor location, leading to misplacement of needles. Also, hard tissues, such as bones in the puncture path might deform the needles, resulting in an inaccurate seed implantation.

Despite the efforts to balance the two groups with the propensity score matching method, there are several limitations of the study. Data from different periods were collected retrospectively in the same center. Plus, 
the increase in dosimetric accuracy may be due to an improvement of skills of the doctors. Moreover, the data on clinical efficacy and side effects were still insufficient. Further follow-up and additional research are needed to investigate whether the increase in dosimetric accuracy can cause the improvement of efficacy and reduction of side effects. Moreover, further large-sample studies are warranted to compare the differences of dosimetry, efficacy, and side effects between CT-guided RIS implantation assisted with and without 3D-PNCT.

\section{Conclusions}

In conclusion, the key to improve efficacy is to improve the accuracy of seed implantation. 3D-PNCT can support the positioning and orientation of the needles. The dose delivered to the tumor was increased, and the "cold" spots of dose were reduced by 3D-PNCT to further improve clinical efficacy. Additional studies shall be conducted for strong evidence of real benefits from the application of 3D-PNCT.

\section{Disclosure}

The authors report no conflict of interest.

\section{References}

1. Zaorsky NG, Davis BJ, Nguyen PL et al. (P034) Evolution of brachytherapy for prostate cancer. Int J Radiat Oncol Biol Phys 2017; 98 .

2. Ji Z, Jiang $Y$, Tian $S$ et al. The effectiveness and prognostic factors of CT-guided radioactive I-125 seed implantation for the treatment of recurrent head and neck cancer after external beam radiation therapy. Int J Radiat Oncol Biol Phys 2019; 103: 638-645.

3. Wang ZM, Lu J, Liu T et al. CT-guided interstitial brachytherapy of inoperable non-small cell lung cancer. Lung Cancer 2011; 74: 253-257.

4. Wang $\mathrm{H}$, Wang J, Jiang $\mathrm{Y}$ et al. The investigation of $125 \mathrm{I}$ seed implantation as a salvage modality for unresectable pancreatic carcinoma. J Exp Clin Cancer Res 2013; 32: 106.

5. Wang JJ, Yuan HS, Li JN et al. Interstitial permanent implantation of 125 I seeds as salvage therapy for re-recurrent rectal carcinoma. Int J Colorectal Dis 2009; 24: 391-399.

6. Huang MW, Liu SM, Zheng L et al. A digital model individual template and CT-guided 125I seed implants for malignant tumors of the head and neck. J Radiat Res 2012; 53: 973-977.

7. Huang W, Lu J, Chen KM et al. Preliminary application of 3D-printed coplanar template for iodine-125 seed implantation therapy in patients with advanced pancreatic cancer. World J Gastroenterol 2018; 24: 5280-5287.

8. Ji Z, Jiang Y, Guo F et al. Safety and efficacy of CT-guided radioactive iodine-125 seed implantation assisted by a 3D printing template for the treatment of thoracic malignancies. J Cancer Res Clin Oncol 2020; 146: 229-236.

9. Liu S, Wang H, Wang C et al. Dosimetry verification of 3D-printed individual template based on CT-MRI fusion for radioactive ${ }^{125} \mathrm{I}$ seed implantation in recurrent high-grade gliomas. J Contemp Brachytherapy 2019; 11: 235-242.

10. Saw CB, Suntharalingam N. Quantitative assessment of interstitial implants. Int J Radiat Oncol Biol Phys 1991; 20: 135-139.

11. van't Riet A, Mak ACA, Moerland MA et al. A conformation number to quantify the degree of conformality in brachyther- apy and external beam irradiation: application to the prostate. Int J Radiat Oncol Biol Phys 1997; 37: 731-736.

12. Yamada $K$, Ishizawa $T$, Niwa $K$ et al. Patterns of pelvic invasion are prognostic in the treatment of locally recurrent rectal cancer. Br J Surg 2001; 88: 988-993.

13. Austin PC. Balance diagnostics for comparing the distribution of baseline covariates between treatment groups in propensity-score matched samples. Statist Med 2009; 28: 3083-3107.

14. Ji Z, Jiang Y, Guo F et al. Dosimetry verification of radioactive seed implantation for malignant tumors assisted by 3D printing individual templates and CT guidance. Appl Radiat Isot 2017; 124: 68-74.

15. Ji Z, Jiang Y, Su L et al. Dosimetry verification of (125)I seeds implantation with three-dimensional printing noncoplanar templates and CT guidance for paravertebral/retroperitoneal malignant tumors. Technol Cancer Res Treat 2017; 16: 1044-1050.

16. Ji Z, H Sun, Y Jiang et al. Comparative study for CT-guided (125)I seed implantation assisted by 3D printing coplanar and non-coplanar template in peripheral lung cancer. J Contemp Brachytherapy 2019; 11: 169-173.

17. Wang $L$, Wang $H$, Jiang $Y$ et al. The efficacy and dosimetry analysis of CT-guided (125) I seed implantation assisted with 3D-printing non-co-planar template in locally recurrent rectal cancer. Radiat Oncol 2020; 15: 179.

18. Han T, Yang X, Xu Y et al. Therapeutic value of 3-D printing template-assisted (125)I-seed implantation in the treatment of malignant liver tumors. Onco Targets Ther 2017; 10: 3277-3283.

19. Keyes M, Morris WJ, Spadinger I et al. Radiation oncology and medical physicists quality assurance in British Columbia Cancer Agency Provincial Prostate Brachytherapy Program. Brachytherapy 2013; 12: 343-355.

20. Zapatero A, Garcia-Vicente F, Martin de Vidales C et al. Long-term results after high-dose radiotherapy and adjuvant hormones in prostate cancer: how curable is high-risk disease? Int J Radiat Oncol Biol Phys 2011; 81: 1279-1285.

21. Stock RG, Stone NN, Tabert A et al. A dose-response study for I-125 prostate implantation. Int J Radiat Oncol Biol Phys 1998; 41: 101-108.

22. Ling CC, Roy J, Sahoo $\mathrm{N}$ et al. Quantifying the effect of dose inhomogeneity in brachytherapy: application to permanent prostatic implant with 125 I seeds. Int J Radiat Oncol Biol Phys 1994; 28: 971-978.

23. Nag S, Beyer D, Friedland J et al. American Brachytherapy Society (ABS) recommendations for transperineal permanent brachytherapy of prostate cancer. Int J Radiat Oncol Biol Phys 1999; 44: 789-799.

24. Kim DWN, Medin PM, Timmerman RD. Emphasis on repair, not just avoidance of injury, facilitates prudent stereotactic ablative radiotherapy. Semin Radiat Oncol 2017; 27: 378-392. 